\title{
Insatisfação com o peso corporal e fatores associados em adolescentes
}

\author{
Body weight dissatisfaction and associated factors among adolescents
}

Giovâni Firpo Del Duca1', Leandro Martin T. Garcia², Thiago Ferreira de Sousa², Elusa Santina A. de Oliveira ${ }^{3}$, Markus Vinicius Nahas ${ }^{4}$

\section{RESUMO}

Objetivo: Estimar a ocorrência de insatisfação com peso corporal e os fatores associados em adolescentes.

Métodos: Estudo transversal, representativo dos escolares de 15 a 19 anos de escolas públicas de Santa Catarina, no ano de 2002. O desfecho do estudo foi a satisfação com o peso corporal, avaliada pela pergunta: "Você está satisfeito(a) com seu peso corporal?”. As variáveis avaliadas foram: sexo, idade, renda familiar, local de residência, estado nutricional, tempo semanal assistindo televisão, tabagismo e autopercepção de saúde. Investigaram-se também os seguintes comportamentos alimentares: tomar remédios para emagrecer, provocar vômitos após as refeições e realizar, no mínimo, três refeições/ dia. Empregou-se regressão multinomial nas análises bruta e ajustada.

Resultados: Dentre os adolescentes avaliados $(n=5.028)$, 16,7\% (IC95\%: 15,7-17,7) estavam insatisfeitos com seu peso e gostariam de aumentá-lo, e 36,2\% (IC95\%: 34,9-37,5) gostariam de diminui-lo. Tomar remédios para emagrecer, provocar vômitos após as refeições e não realizar, no mínimo, três refeições/dia foram mais frequentes entre aqueles que gostariam de reduzir o peso. Após análise ajusta$\mathrm{da}$, os fatores associados ao desejo de aumentar o peso foram: sexo masculino, idade $\geq 17$ anos, residir na zona urbana, não ter excesso de peso e autopercepção de saúde negativa. Já os fatores associados ao desejo de diminuir o peso foram: sexo feminino, residir na zona urbana, ter excesso de peso e uma percepção negativa de saúde.
Conclusões: Encontrou-se alta prevalência de insatisfação com o peso, sendo as variáveis sexo, estado nutricional e autopercepção de saúde os fatores mais fortemente associados a tal comportamento.

Palavras-chave: peso corporal; percepção de peso; imagem corporal; adolescente.

\section{ABSTRACT}

Objective: To assess the prevalence of body weight dissatisfaction among adolescents and the associated factors.

Methods: Population-based cross-sectional study covering a sample of students with ages from 15 to 19 years, attending public high schools in Santa Catarina, Brazil, in the year 2002. The outcome of the study was body weight satisfaction, assessed by the question: "Are you satisfied with your body weight?" The variables analyzed were gender, age, family income, home neighborhood, nutritional status, time of television viewing per week, smoking and self-rated health. Some feeding behaviors were also investigated: taking weight loss medication, vomiting after meals, and having at least three meals per day. A multinomial regression model was used in the crude and adjusted analyses.

Results: Among the adolescents evaluated ( $n=5,028)$, $16.7 \%$ (95\% CI: 15.7-17.7) were unsatisfied and would like to gain weight, and 36.2\% (95\% CI: 34.9-37.5) would like to reduce it. Frequent behaviors among those who wanted to reduce their body weight included: taking weight loss medication,

Endereço para correspondência:

Giovâni Firpo Del Duca

Campus Universitário - Trindade - UFSC

CEP 88040-900 - Florianópolis/SC

E-mail: gfdelduca@gmail.com

Fonte financiadora: Conselho Nacional de Desenvolvimento Científico e Tecnológico (CNPq)

Conflitos de interesse: nada a declarar

Recebido em: 6/11/09 Aprovado em: 15/4/10 
vomiting after meals, and not having at least three meals per day. Adjusted analyses showed that factors associated with the desire to gain weight were: male gender, $\geq 17$ years old, living in the urban area, without overweight, and having a negative selfperception of health. Factors associated with the desire to lose weight included: female gender, living in the urban area, with overweight, and having a negative self-perception of health.

Conclusions: A high prevalence of body weight dissatisfaction was found in this sample, and the factors more associated with this behavior included gender, nutritional status and self-perception of health.

Key-words: body weight; weight perception; body image; adolescent.

\section{Introdução}

A imagem corporal consiste em um constructo multifacetado referente às percepções, pensamentos e sentimentos que o indivíduo possui a respeito de seu corpo ${ }^{(1)}$. Os componentes de caracterização da imagem corporal estão relacionados à percepção do corpo, a partir da avaliação do tamanho e forma corporais, bem como ao conceito do corpo, representado pela autoestima ${ }^{(2)}$.

Dentre as dimensões mensuráveis da imagem corporal, o peso tem recebido foco por parte dos pesquisadores. Grande parte desses estudos é realizada com adolescentes em países desenvolvidos ${ }^{(3-5)}$, sendo escassas as pesquisas em países em desenvolvimento e, particularmente, com adolescentes. Nessa faixa etária, a insatisfação com o peso corporal pode ser internalizada, especialmente por influência dos grupos sociais e dos padrões de beleza divulgados na mídia ${ }^{(6-8)}$.

A insatisfação com o peso corporal constitui-se em um importante preditor de diversos agravos à saúde de adolescentes, como desordens alimentares; jejum prolongado; uso de laxantes e comportamento bulímico ${ }^{(2,9,10)}$; alterações psicológicas, como depressão e baixa autoestima ${ }^{(11)}$; e perda ou ganho de peso por métodos não-saudáveis, como o uso de esteroides para aumento de peso $^{(12,13)}$ e consumo de cigarro para controle de peso ${ }^{(3,14)}$.

Sabe-se que o aumento da prevalência de sobrepeso e obesidade $^{(15)}$, característica da transição nutricional constatada atualmente, é um importante marcador da insatisfação com peso corporal em adolescentes ${ }^{(7,16)}$. Além disso, muitos jovens, embora estejam com peso adequado, referem estar insatisfeitos com seu peso ${ }^{(17)}$. Sendo assim, o objetivo do presente estudo foi estimar a ocorrência de insatisfação com peso corporal e os fatores associados ao desejo de aumento e redução de peso corporal em adolescentes de ambos os sexos, de 15 a 19 anos.

\section{Métodos}

O presente estudo originou-se da pesquisa intitulada "Estilo de Vida e Comportamentos de Saúde dos Jovens Catarinenses", realizado em 2002, em Santa Catarina. O delineamento empregado foi transversal e teve como população alvo os adolescentes de 15 a 19 anos matriculados no ensino médio de escolas públicas do Estado. Na época, Santa Catarina estava dividida em 26 Unidades Regionais de Educação, as quais atendiam um total de 205.543 jovens matriculados no ensino médio, conforme dados da Secretaria Estadual de Educação(18).

O processo amostral por conglomerados foi estratificado de acordo com as cinco regiões geográficas de Santa Catarina (Oeste, Planalto Serrano, Norte, Vale do Itajaí, Litoral e Sul), apresentando como unidades amostrais primárias as escolas e como unidades amostrais secundárias, as turmas. O sorteio das escolas foi proporcional ao tamanho das mesmas, classificando-se as instituições de ensino como sendo de pequeno (<200 alunos), médio (200 a 499 alunos) ou grande porte ( $\geq 500$ alunos). Para o sorteio das turmas, levou-se em consideração a proporcionalidade do número de alunos conforme o turno de estudo (matutino, vespertino e noturno).

Para o cálculo de tamanho amostral, foram utilizados os seguintes parâmetros e estimativas: prevalências dos desfechos de $50 \%$, nível de confiança de $95 \%$, poder de $80 \%$, erro amostral de $2 \%$ e adicional de $25 \%$ para perdas e recusas. Além disso, empregou-se o efeito de delineamento igual a dois, em função da realização da amostragem por conglomerados. O resultado do cálculo gerou o valor da amostra final de 6.000 escolares.

As variáveis dependentes neste estudo são derivadas da percepção de peso corporal, avaliada a partir da seguinte questão: "Você está satisfeito(a) com seu peso corporal?". As opções de resposta possíveis de serem relatadas foram: "sim", "não, gostaria de aumentar" e "não, gostaria de diminuir", correspondendo, respectivamente, às categorias de satisfação com peso corporal, insatisfação com o baixo peso corporal e insatisfação com o excesso de peso corporal. Já as variáveis independentes do estudo foram categorizadas do seguinte modo: sexo (masculino e feminino); idade (15 e 16 e 17 a 19 anos completos); local de residência (zona urbana e zona rural); renda familiar mensal (categorizada em $\leq \mathrm{R} \$$ 500,00; R \$ 501,00 a R \$ 1.000,00 e $\geq \mathrm{R} \$ 1.001,00$ ); estado nutricional (sem excesso e com excesso de peso), a partir da adoção da classificação proposta por Cole et al. ${ }^{(19)}$, que leva em consideração o sexo e faixa etária do adolescente; tabagismo atual (sim ou não); tempo diário assistindo televisão durante o final de semana ( $<4$ e $\geq 4$ horas); e autopercepção de saúde 
(categorizada em positiva, incluindo as respostas "excelente" e "muito boa", e negativa, pelas respostas "regular" e "ruim").

A coleta de dados foi realizada por profissionais e acadêmicos de Educação Física previamente treinados. O instrumento utilizado foi o questionário autoaplicado e com supervisão na própria sala de aula.

A digitação dos dados foi realizada no programa EpiInfo - versão 6.04d (Centers for Disease Control and Prevention, Atlanta, Estados Unidos), com checagem automática de amplitude e consistência. Para a análise dos dados, foi utilizado o programa Statistical Package for the Social Science para Windows, versão 16.0 (SPSS Inc, Chicago, Estados Unidos). Foi empregada a estatística descritiva para o cálculo de frequências absolutas e relativas, médias e desvios padrão (DP). Utilizou-se a regressão multinomial nas análises bruta e ajustada, com resultados expressos em Odds Ratio (OR) e intervalos de confiança de 95\% (IC95\%). Na análise ajustada, foi respeitada a hierarquia entre os possíveis fatores associados com o desfecho ${ }^{(20)}$, adotando-se o seguinte modelo de análise: nível distal - sexo, idade, local de residência, renda familiar mensal; nível intermediário - estado nutricional, nível de atividade física, tabagismo atual, tempo diário assistindo televisão durante a semana escolar; nível proximal - autopercepção de saúde. O efeito de cada exposição sobre o desfecho foi ajustado para as demais variáveis do mesmo nível ou de níveis superiores. Para a modelagem estatística, adotou-se a estratégia de seleção para trás e um nível crítico de $p \leq 0,20$ para permanência no modelo, para controle de confusão.

O estudo foi aprovado pelo Comitê de Ética em Pesquisa da Universidade Federal de Santa Catarina e está de acordo com a resolução 196/96 do Conselho Nacional de Saúde. Os escolares participantes do estudo foram informados sobre os objetivos da pesquisa e o anonimato das informações obtidas.

\section{Resultados}

Do total de 6.000 sujeitos inicialmente incluídos no estudo, $9 \%$ foram perdas ou recusas. Além disso, em função de alguns escolares estarem fora da faixa etária de interesse do estudo (15 a 19 anos), optou-se pela exclusão de 435 indivíduos, sendo a amostra final composta por 5.028 escolares.

A maioria dos participantes eram moças (59,3\%). A idade dos indivíduos variou de 15 a 19 anos, sendo a média de 17,1ะ1,13. Um total de 34,4\% dos adolescentes pertencia a famílias com renda de até $\mathrm{R} \$ 500,00$. Quanto ao local de residência, 3.980 $(79,6 \%)$ adolescentes residiam na zona urbana. A maioria da amostra foi formada por escolares sem excesso de peso $(90,1 \%)$, que assistiam televisão por menos de 4 horas/dia durante a semana escolar $(67,7 \%)$, não-fumantes $(93,2 \%)$ e que relataram perceber sua saúde como positiva $(85,6 \%)$. Mais detalhes de caracterização da amostra são apresentados na Tabela 1.

A prevalência de satisfação com o peso corporal foi de 47,1\% (IC95\%: 45,8-48,5). Os adolescentes insatisfeitos com seu peso e que gostariam de aumentá-lo representaram 16,7\% (IC95\%: 15,7-17,7) da amostra, enquanto aqueles que gostariam de diminui-lo, 36,2\% (IC95\%: 34,9-37,5).

$\mathrm{Na}$ Figura 1, observam-se alguns comportamentos relacionados à alimentação conforme a satisfação com o peso corporal dos escolares investigados. As ações de provocar vômito $(6,2 \%)$ e tomar remédios para emagrecer $(24,2 \%)$ estiveram associadas ao desejo de diminuir o peso. Com relação ao fato de sempre realizar três ou mais refeições/ dia, aqueles que desejavam diminuir o peso adotavam tal comportamento com menor frequência $(51,4 \%)$.

$\mathrm{Na}$ análise bruta do grau de satisfação com o peso corporal (Tabela 2), as variáveis associadas à insatisfação referente ao desejo de aumentar o peso foram: sexo masculino, idade $\geq 17$

Tabela 1 - Descrição dos adolescentes escolares do ensino médio de escolas públicas de Santa Catarina, 2002

\begin{tabular}{|c|c|c|}
\hline Variáveis & $\mathbf{n}$ & $\%$ \\
\hline \multicolumn{3}{|l|}{ Sexo } \\
\hline Feminino & 2.984 & 59,3 \\
\hline Masculino & 2.044 & 40,7 \\
\hline \multicolumn{3}{|l|}{ Faixa etária } \\
\hline 15 e 16 anos & 2.456 & 48,8 \\
\hline 17 a 19 anos & 2.572 & 51,2 \\
\hline \multicolumn{3}{|l|}{ Renda familiar ( $R \$)$} \\
\hline$\leq 500,00$ & 1.697 & 34,4 \\
\hline 501,00 a 1.000 & 1.831 & 37,1 \\
\hline$\geq 1.001,00$ & 1.405 & 28,5 \\
\hline \multicolumn{3}{|l|}{ Local de residência } \\
\hline Zona rural & 1.020 & 20,4 \\
\hline Zona urbana & 3.980 & 79,6 \\
\hline \multicolumn{3}{|l|}{ Estado nutricional } \\
\hline Sem excesso de peso & 4.444 & 90,1 \\
\hline Com excesso de peso & 489 & 9,9 \\
\hline \multicolumn{3}{|l|}{ Tempo de TV na semana* } \\
\hline$<4$ horas & 2.972 & 67,7 \\
\hline$\geq 4$ horas & 1.419 & 32,3 \\
\hline \multicolumn{3}{|l|}{ Tabagismo atual } \\
\hline Não & 4.634 & 93,2 \\
\hline Sim & 336 & 6,8 \\
\hline \multicolumn{3}{|l|}{ Autopercepção de saúde } \\
\hline Positiva & 4.242 & 85,6 \\
\hline Negativa & 711 & 14,4 \\
\hline
\end{tabular}

* Variável com maior número de valores ignorados ( $n=637$ ). 


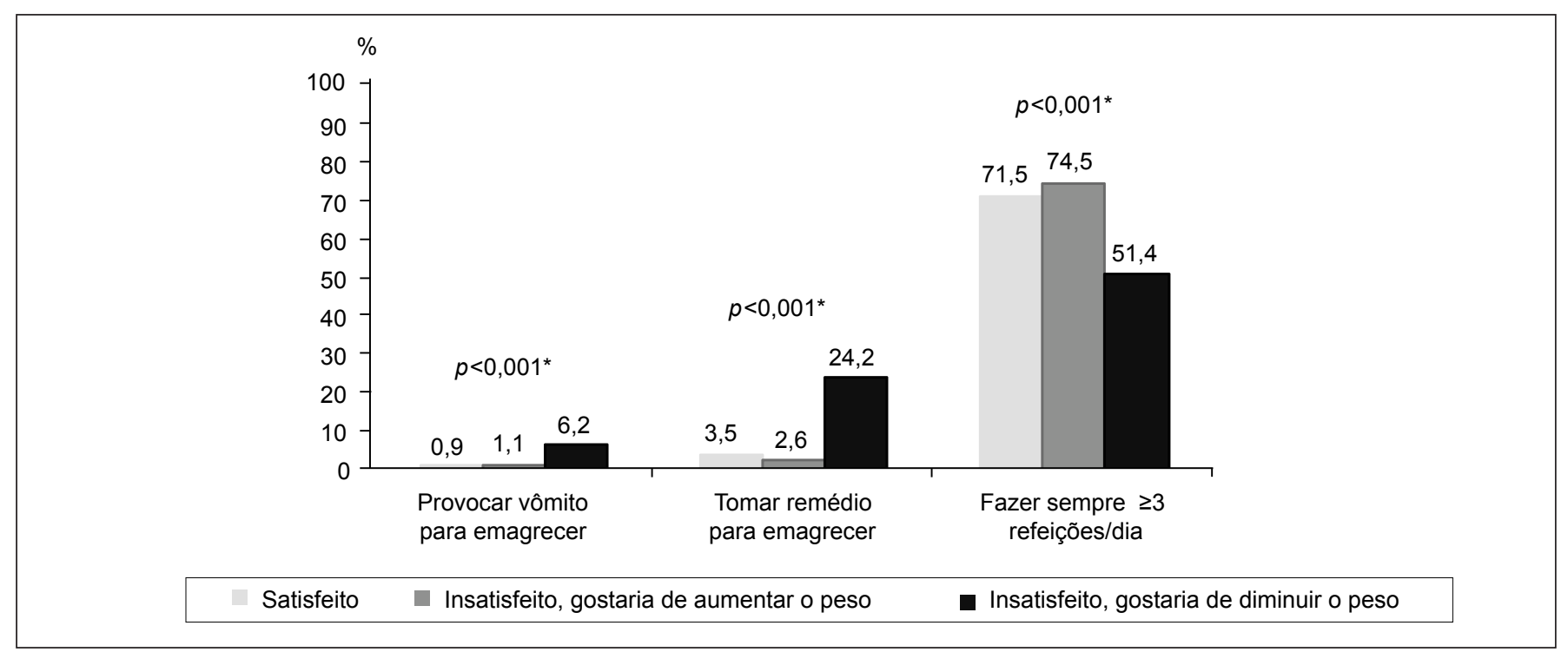

Figura 1 - Prevalência de comportamentos relacionados à alimentação conforme a satisfação com o peso corporal em adolescentes do ensino médio de escolas públicas de Santa Catarina, 2002. *Qui-quadrado de Pearson.

Tabela 2 - Prevalência, análise bruta e ajustada da satisfação com peso corporal em adolescentes do ensino médio de escolas públicas de Santa Catarina, 2002

\begin{tabular}{|c|c|c|c|c|c|c|c|}
\hline \multirow{2}{*}{ Variáveis } & \multirow{2}{*}{$\begin{array}{c}\text { Satisfeito } \\
\%\end{array}$} & \multicolumn{3}{|c|}{$\begin{array}{c}\text { Insatisfeito } \\
\text { (gostaria de aumentar o peso) }\end{array}$} & \multicolumn{3}{|c|}{$\begin{array}{c}\text { Insatisfeito } \\
\text { (gostaria de diminuir o peso) }\end{array}$} \\
\hline & & $\%$ & $\begin{array}{l}\text { OR bruta } \\
\text { (IC95\%) }\end{array}$ & $\begin{array}{l}\text { OR ajustada } \\
\text { (IC95\%) }\end{array}$ & $\%$ & $\begin{array}{l}\text { OR bruta } \\
\text { (IC95\%) }\end{array}$ & $\begin{array}{l}\text { OR ajustada } \\
\text { (IC95\%) }\end{array}$ \\
\hline \multicolumn{8}{|l|}{ Sexo } \\
\hline Feminino & 40,3 & 10,9 & 1,00 & 1,00 & 48,8 & $3,95(3,42-4,55)$ & $3,99(3,45-4,62)$ \\
\hline Masculino & 57,3 & 25,2 & $1,62(1,38-1,90)$ & $1,65(1,40-1,95)$ & 17,6 & 1,00 & 1,00 \\
\hline \multicolumn{8}{|l|}{ Faixa etária } \\
\hline 15 e 16 anos & 47,2 & 14,8 & 1,00 & 1,00 & 38,0 & 1,00 & 1,00 \\
\hline 17 a 19 anos & 47,1 & 18,5 & $1,25(1,06-1,46)$ & $1,21(1,03-1,43)$ & 34,4 & $0,91(0,80-1,03)$ & $0,97(0,85-1,11)$ \\
\hline \multicolumn{8}{|l|}{ Renda familiar (R\$) } \\
\hline$\leq 500,00$ & 46,3 & 15,8 & $0,98(0,80-1,20)$ & $1,09(0,88-1,35)$ & 37,8 & $1,06(0,91-1,24)$ & $0,97(0,82-1,14)$ \\
\hline 501,00 a $1.000,00$ & 47,4 & 17,6 & $1,06(0,87-1,30)$ & $1,11(0,91-1,36)$ & 35,0 & $0,96(0,82-1,12)$ & $0,90(0,77-1,06)$ \\
\hline$\geq 1.001,00$ & 47,2 & 16,5 & 1,00 & 1,00 & 36,3 & 1,00 & 1,00 \\
\hline \multicolumn{8}{|l|}{ Local de residência } \\
\hline Zona rural & 53,0 & 14,7 & 1,00 & 1,00 & 32,4 & 1,00 & 1,00 \\
\hline Zona urbana & 45,6 & 17,2 & $1,36(1,11-1,67)$ & $1,46(1,18-1,80)$ & 37,2 & $1,33(1,14-1,56)$ & $1,27(1,08-1,50)$ \\
\hline \multicolumn{8}{|l|}{ Estado nutricional } \\
\hline Sem excesso de peso & 50,0 & 18,0 & $1,40(0,90-2,17)$ & $1,58(0,99-2,53)$ & 32,0 & 1,00 & 1,00 \\
\hline Com excesso de peso & 20,9 & 5,4 & 1,00 & 1,00 & 73,8 & $5,52(4,38-6,96)$ & $10,15(7,69-13,39)$ \\
\hline \multicolumn{8}{|l|}{ Tempo de TV na semana } \\
\hline$<4$ horas & 48,0 & 16,6 & 1,00 & 1,00 & 35,4 & 1,00 & 1,00 \\
\hline$\geq 4$ horas & 45,3 & 16,7 & $1,06(0,89-1,27)$ & $1,09(0,91-1,32)$ & 38,0 & $1,14(0,99-1,31)$ & $1,10(0,94-1,29)$ \\
\hline \multicolumn{8}{|l|}{ Tabagismo atual } \\
\hline Não & 47,6 & 16,6 & 1,00 & 1,00 & 35,8 & 1,00 & 1,00 \\
\hline Sim & 41,3 & 16,2 & $1,13(0,81-1,57)$ & $1,00(0,70-1,44)$ & 42,5 & $1,37(1,07-1,75)$ & $1,32(0,98-1,77)$ \\
\hline \multicolumn{8}{|l|}{ Autopercepção de saúde } \\
\hline Positiva & 49,4 & 16,0 & 1,00 & 1,00 & 34,6 & 1,00 & 1,00 \\
\hline Negativa & 32,3 & 21,1 & $2,02(1,61-2,53)$ & $2,28(1,80-2,89)$ & 46,6 & $2,06(1,71-2,47)$ & $1,61(1,32-1,97)$ \\
\hline
\end{tabular}

OR: Odds Ratio; IC95\%: intervalo de confiança de $95 \%$. 
anos, residir na zona urbana e ter autopercepção de saúde negativa. $\mathrm{Na}$ análise ajustada, a probabilidade de relatar a insatisfação referente ao desejo de aumento do peso corporal foi maior entre os rapazes (OR: 1,65; IC95\%: 1,40-1,95), escolares com idade $\geq 17$ anos (OR: 1,21; IC95\%: 1,031,43), os que residiam na zona urbana (OR: 1,46; IC95\%: $1,18-1,80)$ e com autopercepção de saúde negativa (OR: 2,28; IC95\%: 1,80-2,89).

$\mathrm{Na}$ análise bruta referente à insatisfação com o peso corporal ligada ao desejo de diminuir o peso (Tabela 2), as variáveis que se associaram ao desfecho foram: sexo feminino, residir na zona urbana, ter excesso de peso, ser tabagista e ter uma percepção de saúde negativa. $\mathrm{Na}$ análise ajustada, permaneceram associadas a tal insatisfação as seguintes variáveis: sexo feminino (OR 3,99; IC95\% 3,45-4,62), residir na zona urbana (OR 1,27; IC95\% 1,08-1,50), ter excesso de peso (OR 10,15; IC95\% 7,69-13,39) e perceber sua própria saúde como negativa (OR 1,61; IC95\% 1,32-1,97).

\section{Discussão}

As principais contribuições do presente estudo residem na estimativa de insatisfação com o peso corporal e na investigação dos fatores demográficos, socioeconômicos, comportamentais e relacionados à saúde associados a tal insatisfação em adolescentes. A carência de estudos em países em desenvolvimento, a análise de importantes diferenças entre moças e rapazes quanto à insatisfação com o peso corporal, bem como a descrição de comportamentos alimentares de risco adotados por jovens que desejam diminuir o peso precisam ser mais bem considerados por profissionais de saúde e educação que trabalham com indivíduos nessa faixa etária.

No presente estudo, dentre os adolescentes avaliados, 16,7\% (IC95\%: 15,7-17,7) estavam insatisfeitos com seu peso, pois gostariam de aumentá-lo, e 36,2\% (IC95\%: 34,9-37,5) gostariam de diminui-lo. Esses dados estão relativamente próximos daqueles encontrados em Porto Alegre, Rio Grande do Sul ${ }^{(21)}$. Guardadas as diferenças de faixa etária e a restrição da investigação ao sexo feminino, os autores notaram que $13,0 \%$ das mulheres sentiam-se magras e $37,8 \%$ sentiam-se gordas.

$\mathrm{Na}$ China, realizou-se pesquisa ${ }^{(22)} \mathrm{com} 6.863$ adolescentes com grau de escolaridade de 7 a 11 anos. Os resultados encontrados apontaram que $28,1 \%$ dos adolescentes sentiamse magros demais e um total de $38,7 \%$ percebiam-se como relativamente ou muito pesados - valores próximos aos encontrados nesta análise com adolescentes catarinenses.
Recente estudo ${ }^{(4)}$ desenvolvido em 35 países e regiões da Europa, Estados Unidos e Canadá, com apoio da Organização Mundial da Saúde, investigou o desejo de perder peso entre adolescentes. As mais altas ocorrências foram encontradas na República Checa, onde 48,0\% dos rapazes e moças relataram estar fazendo dieta ou algo similar para perder peso, seguida pela Itália (47,6\%), Estados Unidos (44,4\%), País de Gales (43,6\%), Grécia (43,6\%) e Croácia (42,7\%). Vale destacar que as disparidades das prevalências de excesso de peso entre países desenvolvidos e em desenvolvimento podem contribuir para as diferentes ocorrências de insatisfação com o peso corporal.

Estudos recentes ${ }^{(4,23)}$ sobre insatisfação com peso corporal têm demonstrado diferentes resultados quando foram comparados rapazes e moças. Enquanto elas buscam preferencialmente um corpo mais magro ${ }^{(4)}$, eles optam, em sua maioria, por um corpo maior e mais pesado ${ }^{(23)}$, certamente no sentido de ganho muscular e não de maior acúmulo de gordura corporal.

Em nosso estudo, com relação ao desejo de aumentar o peso, rapazes relataram mais frequentemente do que as moças (OR: 1,65; IC95\%: 1,40-1,95) essa opção de resposta quando indagados sobre sua satisfação com o peso corporal. Entretanto, com relação ao desejo de diminuir o peso, os resultados foram ainda mais expressivos, porém, dessa vez, com maior prevalência entre as moças (OR: 3,99; IC95\%: 3,45-4,62). Uma possível explicação relaciona-se ao fato de que os homens, especialmente na adolescência, não sofrem tanta pressão social, apresentando uma melhor aceitação de seu o corpo ${ }^{(24)}$. Estudo longitudinal apontou que a insatisfação corporal sofre influência das conversas sobre aparência física com amigos e das comparações sociais ${ }^{(25)}$. No entanto, apesar de as preocupações com relação à aparência física serem distintas entre os sexos, na adolescência, rapazes e moças parecem valorizar o peso corporal, o que é um importante indicativo desse comportamento ${ }^{(25)}$.

$\mathrm{Na}$ presente análise, o aumento da idade esteve associado ao desejo de aumentar o peso corporal, mas não ao desejo de diminui-lo. Estudo longitudinal ${ }^{(16)}$ realizado com mais de 2.000 escolares dos Estados Unidos investigou a mudança da satisfação com a imagem corporal em adolescentes. Em cinco anos de acompanhamento, a satisfação com a imagem corporal declinou significativamente, com exceção do grupo de moças com maior idade. Há, portanto, indícios de que a prevenção primária a respeito da insatisfação com a imagem corporal deve ocorrer logo no início da adolescência, idealmente antes da satisfação corporal começar a declinar ${ }^{(16,23)}$. 
Com relação ao local de residência, estudo realizado em dois municípios do Estado do Rio Grande do Sul ${ }^{(26)}$ encontrou que escolares residentes na zona urbana tinham maior probabilidade de estarem insatisfeitos com sua imagem corporal. Apesar de o foco do presente estudo ser a insatisfação com o peso corporal, e não propriamente a imagem corporal, tais temáticas guardam forte relação. Em nosso trabalho, escolares residentes na zona urbana mostraram-se mais insatisfeitos com seu peso corporal, tanto em relação ao desejo de aumentar como de diminuir o peso. Algumas possibilidades para tal resultado são as possíveis diferenças entre jovens da zona urbana e rural quanto ao acesso e exposição aos meios de comunicação (importantes disseminadores de padrões estéticos), bem como as marcantes diferenças de condições, estilos e percepções de vida e bem-estar geral.

Grande corpo de evidências científicas com delineamento transversal ${ }^{(4,26)}$ e longitudinal ${ }^{(16,25)}$ aponta a relação entre elevados índices de massa corpórea e insatisfação com peso e imagem corporal. Em nossas análises, o excesso de peso em adolescentes que gostariam de diminuí-lo foi significativo para a insatisfação com o peso corporal.

Comportamentos relacionados à alimentação também guardam importante relação com a insatisfação corporal. As desordens alimentares em adolescentes que buscam perder peso podem ser exemplificadas pelo jejum prolongado, uso de laxantes e comportamentos bulímicos e anoréxicos ${ }^{(2,9,10)}$. Na presente análise, além de as ações de provocar vômitos e tomar medicamentos para emagrecer serem mais frequentes entre os adolescentes que buscam diminuir o peso, estes relataram, com menor frequência, fazerem três ou mais refeições/dia.

O desejo de adolescentes de mudar seu estado nutricional fica evidente, remetendo a importantes considerações sobre a divulgação do sobrepeso/obesidade como problemas de Saúde Pública. Não basta apenas o conhecimento dos riscos do excesso de peso para a saúde, é preciso que políticas públicas voltadas aos adolescentes possam intervir efetivamente nesse quadro, seja por meio da educação no ambiente escolar e familiar ou pela implementação de estratégias relacionadas à promoção de alimentação saudável e atividades físicas, duas importantes vertentes que, quando intimamente ligadas, produzem resultados satisfatórios na redução de peso corporal.

Em nossas análises, a autopercepção de saúde, mesmo após controle para variáveis demográficas, econômicas e comportamentais, foi uma das exposições mais associadas à satisfação com o peso corporal nos adolescentes avaliados. Ou seja, indivíduos com autopercepção negativa de saúde apresentaram maiores ocorrências de insatisfação com o peso corporal. A literatura também aponta que adolescentes com percepção negativa de saúde apresentam maior consumo de cigarro e possuem maior nível de estresse ${ }^{(27)}$. Recente estudo ${ }^{(28)}$ realizado com adolescentes palestinos encontrou que reclamações ligadas à saúde geral, como pior autopercepção de saúde e doenças psicológicas e somáticas, estão associadas à maior insatisfação com o peso corporal. Tal relação pode ser explicada pelo fato de que adolescentes têm na saúde física a principal essência da representação de sua saúde ${ }^{(29)}$, embora se saiba que tal conceito envolve outras dimensões de ordem psicológica e social. Enfim, a insatisfação com o peso corporal e os diversos comportamentos de risco adotados por adolescentes com autopercepção de saúde negativa pode trazer sérios prejuízos à saúde e à qualidade de vida desses indivíduos.

O tabagismo ${ }^{(3,28)}$ e o hábito de ver televisão ${ }^{(6,28)}$ são comportamentos frequentemente estudados quando o alvo está na insatisfação com o peso corporal de adolescentes. $\mathrm{O}$ fato de ver televisão não tem sido associado à maior insatisfação com peso corporal em adolescentes ${ }^{(28)}$, apesar de ser um caminho informativo acerca dos padrões estéticos corporais. Entretanto, o tabagismo é apontado como um hábito preocupante na busca pela perda de peso ${ }^{(3)}$. No presente estudo, o efeito bruto do tabagismo sobre a insatisfação com o peso corporal não foi confirmado na análise ajustada, embora tenha ficado no limiar da significância estatística para os adolescentes que gostariam de perder peso.

Considerável proporção de adolescentes de Santa Catarina mostrou-se insatisfeita com seu peso corporal. Dentre os fatores associados investigados, o sexo, o estado nutricional e a autopercepção de saúde foram as variáveis mais fortemente relacionadas à insatisfação com o peso. Além disso, comportamentos alimentares de risco, como as ações de provocar vômitos após as refeições, tomar medicamentos para emagrecer e não fazer um número mínimo adequado de refeições diárias reforçam a importância da abordagem sobre o assunto por políticas públicas de saúde e educação. Nesse sentido, a escola parece ser um importante local para o repasse de informações e prestação de esclarecimentos sobre os prejuízos que a insatisfação com a imagem e peso corporal, bem como comportamentos alimentares de risco, podem trazer à saúde. 


\section{Referências bibliográficas}

1. Cash TF, Pruzinsky T. Body images: development, deviance and change. New York: The Guilford Press; 1990.

2. Waller $\mathrm{G}$, Hodgson $\mathrm{S}$. Body image distortion in anorexia and bulimia nervosa: the role of perceived and actual control. J Nerv Ment Dis 1996;184:213-9.

3. Fulkerson JA, French SA. Cigarette smoking for weight loss or control among adolescents: gender and racial/ethnic differences. J Adolesc Health 2003;32:306-13.

4. Al Sabbah H, Vereecken CA, Elgar FJ, Nansel T, Aasvee K, Abdeen Z et al. Body weight dissatisfaction and communication with parents among adolescents in 24 countries: international cross-sectional survey. BMC Public Health 2009;9:52.

5. Cinelli RL, O'Dea JA. Body image and obesity among Australian adolescents from indigenous and Anglo-European backgrounds: implications for health promotion and obesity prevention among Aboriginal youth. Health Educ Res 2009;24:1059-68.

6. Felts WM, Parrillo AV, Chenier T, Dunn P. Adolescents' perceptions of relative weight and self-reported weight-loss activities: analysis of 1990 YRBS (Youth Risk behavior Survey) national data. J Adolesc Health 1996;18:20-6.

7. Conti MA, Frutuoso MFP, Gambardella AMD. Obesity and body dissatisfaction amongst adolescents. Rev Nutr 2005;18:491-7.

8. Borzekowski DL, Bayer AM. Body image and media use among adolescents. Adolesc Med Clin 2005;16:289-313.

9. Neumark-Sztainer D, Paxton SJ, Hannan PJ, Haines J, Story M. Does body satisfaction matter? Five-year longitudinal associations between body satisfaction and health behaviors in adolescent females and males. J Adolesc Health 2006;39:244-51.

10. Janssen I, Craig WM, Boyce WF, Pickett W. Associations between overweight and obesity with bullying behaviors in school-aged children. Pediatrics 2004;113:1187-94.

11. Needham BL, Crosnoe R. Overweight status and depressive symptoms during adolescence. J Adolesc Health 2005;36:48-55.

12. Bahrke MS, Yesalis CE, Kopstein AN, Stephens JA. Risk factors associated with anabolic-androgenic steroid use among adolescents. Sports Med 2000;29:397-405.

13. Cohane GH, Pope HG Jr. Body image in boys: a review of the literature. Int J Eat Disord 2001;29:373-9.

14. Strauss RS, Mir HM. Smoking and weight loss attempts in overweight and normal-weight adolescents. Int J Obes Relat Metab Disord 2001;25:1381-5.

15. Crothers LM, Kehle TJ, Bray MA, Theodore LA. Correlates and suspected causes of obesity in children. Psychology in the Schools 2009;46:787-96.
16. Eisenberg ME, Neumark-Sztainer D, Paxton SJ. Five-year change in body satisfaction among adolescents. J Psychosom Res 2006;61:521-7.

17. Ojala K, Vereecken C, Välimaa R, Currie C, Villberg J, Tynjälä J et al. Attempts to lose weight among overweight and non-overweight adolescents: a crossnational survey. Int J Behav Nutr Phys Act 2007;4:50.

18. Instituto Nacional de Estudos e Pesquisas Educacionais Anísio Teixeira (Inep) [homepage na Internet]. Sistema de consulta a matrícula do censo escolar - 1997/2010 [cited 2000 July 2000]. Available from: http://www.inep.gov.br/ basica/censo/Escolar/Matricula/

19. Cole TJ, Bellizzi MC, Flegal KM, Dietz WH. Establishing a standard definition for child overweight and obesity worldwide: international survey. BMJ 2000;320:1240-3.

20. Victora CG, Huttly SR, Fuchs SC, Olinto MT. The role of conceptual frameworks in epidemiological analysis: a hierarchical approach. Int $\mathrm{J}$ Epidemiol 1997;26:224-7.

21. Nunes MA, Olinto MT, Barros FC, Camey S. Influence of body mass index and body weight perception on eating disorders symptoms. Rev Bras Psiquiatr 2001;23:21-7.

22. Xie B, Chou CP, Spruijt-Metz D, Reynolds K, Clark F, Palmer PH et al. Weight perception and weight-related sociocultural and behavioral factors in Chinese adolescents. Prev Med 2006;42:229-34.

23. Vilela JE, Lamounier JA, Dellaretti Filho MA, Barros Neto JR, Horta GM. Eating disorders in school children. J Pediatr (Rio J) 2004;80:49-54.

24. Graham MA, Eich C, Kephart B, Peterson D. Relationship among body image, sex, and popularity of high school students. Percept Mot Skills 2000;90:118793.

25. Carlson Jones D. Body image among adolescent girls and boys: a longitudinal study. Dev Psychol 2004;40:823-35.

26. Triches RM, Giugliani ER. Body dissatisfaction in school children from two cities in the South of Brazil. Rev Nutr 2007;20:119-28.

27. Branco LM, Hilário MO, Cintra IP. Percepção e satisfação corporal em adolescentes e a relação com seu estado nutricional. Rev Psiquiatr Clín (São Paulo) 2006;33:292-6

28. Al Sabbah H, Vereecken C, Abdeen Z, Coats E, Maes L. Associations of overweight and of weight dissatisfaction among Palestinian adolescents: findings from the national study of Palestinian schoolchildren (HBSCWBG2004). J Hum Nutr Diet 2009;22:40-9.

29. Wade TJ, Vingilis $E$. The development of self-rated health during adolescence: an exploration of inter- and intra-cohort effects. Can J Public Health 1999;90: 90-4. 